\title{
SCLEROMA: AN ETIOLOGICAL STUDY
}

\author{
By Milton GJELhaUg LEVINE, ROBERT E. HOYT, and JOHN E. PETERSON \\ (From the Institute of Experimental Medicine and the Department of Medicine, College of \\ Medical Evangelists, Los Angeles, California)
}

(Received for publication September 28, 1946)

Rhinoscleroma was discovered in 1870 by von Hebra (1) who considered it an unusual form of skin cancer. Geber (2) later characterized the lesion as a chronic inflammatory process accompanied by perivascular cellular infiltration and connective tissue proliferation. Mikulicz (3) clearly described the histological picture including the diagnostic "foam" or "lace" cells which have been named after him.

Since the disease is not confined to the nose as von Hebra originally assumed in using the prefix "rhino," the more inclusive term scleroma was officially adopted at the Second International Congress of Otorhino-laryngology (4) held in Madrid in 1932.

\section{PATHOLOGY}

Scleroma is a disease which clinically is well defined. It is a granulomatous lesion primarily localized in the upper respiratory tract. Secondarily it may appear about the pyriform aperture of the vestibule of the nose. In the respiratory tract, scleroma is most often localized in the anterior portion of the nose, in the vestibule or the posterior nares. In the larynx, the lesions are found usually in the subglottal space.

The pharynx and throat are the seat of lesions in a third of the cases. These are found in the mesopharynx, the posterior surface of the soft palate, the uvula, the pillars, and the lateral portion of the posterior wall. The trachea is occasionally involved.

The gross lesion most frequently described is the circumscribed nodular type which may appear as a single nodule or in groups. Each nodule may appear as a single nodule or in groups. Each nodule may vary in size from 1 to $10 \mathrm{~mm}$. Their consistency varies; the early infiltrates are soft while those of longer standing are harder.

Histologically, the picture is that of a granuloma. The ciliated epithelium is transformed into stratified pavement cells. In the deeper layers of the cornea, the protoplasm becomes more dispersed and partially disappears; vacoules and pyknotic nuclei are seen. Perivascular infiltration is found in which the Mikulicz cell stands out.

Clinically, the nodular type is easily recognized. However, one may assume the existence of a prenodular stage which is less likely to be recognized since there have been no accepted specific tests for the disease. Szmurlo (5) has actually described this form of scleroma as the "diffuse" stage. This first "appears as atrophic rhinitis characterized by atrophy of the nasal mucosa with abundant secretion and gray crusts on the surface."

The clinical course of the disease is benign, the onset is insidious, and the duration may be from twenty to thirty years. There is little effect on the general health except for the mechanical obstruction of the air passages which may in extreme cases lead to asphyxia.

\section{INCIDENCE}

Scleroma was once thought to be confined to Central and Southeastern Europe, but it is now recognized more often and is generally acknowledged as world-wide in occurrence. In a comprehensive report, Cunning and Guerry (6) in 1942 reported that 102 cases had been described to that date in the American and Canadian literature. Streit (7) contends that an endemic area exists in Central Europe especially Poland. Lasagna (8) feels that it is becoming more common in Italy, and there is some evidence that the disease is seen frequently in Central and South America.

ETIOLOGY

The problems of scleroma are clouded by the confusion associated with its etiology. In 1882, von Frisch (9) described an organism which he asserted to be the cause of the disease. Due to inadequate morphological studies inherent in the embryonic state of the science of bacteriology and due to the lack of an experimental lesion in 
animals resembling the disease in human beings, Frisch's organism was not universally accepted. The belief expressed by the American, Perkins (10), that the scleroma bacillus was not related to the disease, has been echoed periodically since then, and the current opinion may be seen in the comments of two outstanding bacteriological texts. Gay (11) states, " $K$. rhinoscleromatis bears some as yet obscure relationship with the disease rhinoscleroma. It appears, however, commonly to be a secondary invader in the lesions characteristic of the disease." Wilson and Miles (12) state, "There is very little evidence to show that this organism is primarily responsible for it. There is no means by which it can be distinguished with certainty from other members of the capsulated group; and since we know that members of this group may be present in the nose of healthy persons, it is difficult to prove that they play any part in production of rhinoscleroma. The probability is that they are mere secondary invaders, which grow freely in the nose of patients suffering from the disease."

Much of the etiological difficulty encountered is due to the alleged presence of the scleroma bacillus or related organisms in normal throats. To test this hypothesis, we must establish a criterion for the characteristics of the scleroma bacillus, then we must study its occurrence in the normal throat. The very early literature must be discarded since the required facts about the related Friedlander bacillus and the coli-aerobacter group were not available to these first investigators. There is, however, a remarkable uniformity which has been overlooked in the description of the rhinoscleroma bacillus by a number of reliable investigators in recent years. Figi and Thompson (13) in 1928, described 3 strains isolated from patients at the Mayo Clinic. Morris and Julianelle (14), apparently unaware of Thompson's report, studied 9 strains from cases in Sumatra and this country. Both of these workers found their strains to ferment glucose, maltose, and mannite with the production of acid only. Sucrose was fermented slowly by some strains, lactose never. Indole was not formed, nitrate was reduced. Other reactions are noted but are not essential to the differential identification of the organism. Gonsiorowski and Meisel (15), two
TABLE I

Cultural characteristics of scleroma strains

\begin{tabular}{l|c|c|c|c|c|c|c}
\hline \hline Strain & Mannite & Maltose & Dextrose & Lactose & Sucrose & Nitrate & Indole \\
\hline 1L & A & A & A & - & A & + & - \\
2F & A & A & A & - & - & + & - \\
3J & A & A & A & - & - & + & - \\
4M & A & A & A & - & - & + & - \\
5R & A & A & A & - & - & + & - \\
6C & A & A & A & - & - & + & - \\
7CA & A & A & A & - & - & + & - \\
8T & A & A & A & - & - & + & - \\
\hline
\end{tabular}

Polish workers with a large number of freshly isolated strains at their disposal, reported the fermentation of glucose (acid only) but not of lactose, the only sugars tested.

We have isolated seven strains from cases to be described later in this paper and have obtained one culture from the American Type Culture Collection. The results of cultured studies on these organisms are given in Table I.

From the above it is evident that there is a remarkable uniformity in the reactions of our organisms identical with those obtained by the workers mentioned above. We feel that the characteristics described in Table $I$, including the variable, slow fermentation of sucrose should be considered specific for these organisms. If we include the colony characteristics (large translucent, mucoid, coalescing colonies on blood agar, nutrient agar, and E. M. B. medium), we have a sufficient differential description to identify this organism.

No other related gram negative rod found on the human being has been reported which gives the same pattern of reactions. This is true even of the variable Friedlander group. Julianelle (14), who has done most of the work on the classification of this genus, found no strain in any of the groups A, B, C or the unclassified Friedlander group to give reactions similar to those described for the rhinoscleroma bacillus. We have studied strains of the Friedlander bacillus obtained from the American Type Culture Collection, and find no resemblance of any of these to the scleroma organism (Table II).

From the above and from Julianelle's findings, we concluded that it should be possible to separate the mucoid Friedlander from the mucoid scleroma bacillus. 
TABLE II

Cultural characteristics of Friedlander strains

\begin{tabular}{l|c|c|c|c|c|c|c}
\hline \hline Strain & Mannite & Maltose & $\begin{array}{c}\text { Dex- } \\
\text { trose }\end{array}$ & Lactose & Sucrose & Nitrate & Indole \\
\hline 132 & AG & AG & AG & AG & AG & + & - \\
8044 A & AG & AG & AG & AG & AG & + & - \\
4727 & AG & AG & AG & AG & AG & + & - \\
8045 A & AG & AG & AG & AG & AG & + & - \\
8046 A & AG & AG & AG & AG & AG & + & - \\
6540 & AG & AG & AG & AG & AG & + & - \\
7380 B & A & A & A & A & A & + & - \\
9997 & A & A & A & A & A & + & - \\
4208 & A & A & A & A & A & + & - \\
\hline
\end{tabular}

We next tested the assumption that the scleroma bacillus is found in normal throats. A total of 204 normal individuals and 63 with various nose and throat ailments were cultured. The procedure used was found to the satisfactory in the isolation of the rhinoscleroma organisms in the known infected cases. Nose and throat swabs were streaked on blood agar and E. M. B. in all infected individuals. Since the results on the blood agar and E. M. B. were similar, we employed only the latter in our studies of the normal, and the nose and throat cases.

Gram negative organisms (which morphologically are related to the scleroma bacillus) in the nose and throat that will grow on E. M. B. include members of the coli-aerogenes and Friedlander groups, Proteus, Pseudomonas and Alkaligenes. Most noses and throats did not show a sufficient number of any of these to give a positive

TABLE III

Cultural characteristics of gram negative bacilli isolated from normal individuals or nose and throat cases

\begin{tabular}{|c|c|c|c|c|c|c|c|}
\hline annite & Maltose & $\begin{array}{l}\text { Dex- } \\
\text { trose }\end{array}$ & Lactose & Sucrose & Nitrate & Indole & $\begin{array}{c}\text { Number } \\
\text { of } \\
\text { strains }\end{array}$ \\
\hline AG & AG & AG & AG & AG & + & - & 13 \\
\hline & & & & & + & + & \\
\hline AG & A & A & $\mathrm{AC}$ & AG & - & - & 1 \\
\hline AG & & A & & AG & + & + & 1 \\
\hline AG & A & $\mathrm{AC}$ & AG & & + & + & 2 \\
\hline & & & & AG & + & - & 1 \\
\hline Au & $\mathrm{AC}$ & Af & A & $\mathrm{AG}$ & + & - & 1 \\
\hline $\mathrm{AG}$ & $\mathrm{A}$ & A & A & - & + & \pm & 1 \\
\hline $\mathrm{AC}$ & $\begin{array}{l}\mathrm{A} \\
\mathrm{AC}\end{array}$ & $\begin{array}{l}\mathrm{AG} \\
\mathrm{AG}\end{array}$ & $=$ & $\bar{Z}$ & + & $\bar{t}$ & $\begin{array}{l}2 \\
1\end{array}$ \\
\hline & A & A & A & A & + & - & 1 \\
\hline & I & $\stackrel{A}{A}$ & - & - & $\bar{t}$ & $\overline{+}$ & 1 \\
\hline & - & A & $\overline{-}$ & $\overline{-}$ & $t$ & $t$ & 1 \\
\hline & - & A & - & 1 & $\bar{\tau}$. & - & 4 \\
\hline & & A & & - & + & - & 1 \\
\hline
\end{tabular}

culture. Of a total of 267 individuals tested, only 34 gave positive cultures; the results of cultural studies of these organisms are presented in Table III.

Although we have not as yet speciated the above strains, it is obvious that none has the pattern we have found to be characteristic of the scleroma bacillus. Further work is in progress to name these organisms as to species.

\section{SEROLOGICAL STUDIES}

In order further to relate the organism to the disease in question, serological studies should be of value. There are many references in the literature to such studies. As is true of the cultural studies, the early serological reports are confusing, and for the same reasons mentioned previously. However, more recent studies, if correlated, certainly are not to be disregarded. Goldzieher and Neuber (16), Brault and Masselot (17), and Quast (18) have all agreed that serum from patients with scleroma or from rabbits immunized with the organism is capable of fixing complement. Fitzgerald (19) showed that a rabbit immune serum agglutinated specifically the decapsulated organism. Prasek and Prica (20) and Morris and Julianelle (14) have both demonstrated the specific antigenicity of the organism.

We have employed the complement fixation test in our studies. Seven patients suffering from scleroma have given positive reactions with antigen consisting of heatkilled $\left(65^{\circ} \mathrm{C}\right.$. for 30 minutes) organisms grown on nutrient agar slants.

Over 500 sera from normal patients and from individuals with an assortment of other diseases have been tested with only occasional positive reactions. However, we find such "false positive reactions" to be a function of the concentration of antigen and antiserum. We are now in the process of standardizing the procedure to obtain maximum accuracy. Details of the test will be published elsewhere.

\section{CASE STUDIES}

One further controversial question concerning scleroma has been its contagiousness. Its endemic nature has been suggested previously. Familial occurrence would indicate an infectious etiology. Lasagna (8) Robertson and Secretan (21), and Gerber (22) have reported duplicate cases occurring in the same family. We have studied seven cases, six of which have occurred in the same family.

Case 1. L. M., male, age 20, of Mexican descent, was referred to the clinic because of dyspnea and a cough productive of a thick yellow mucoid material. 
About one year ago he began to notice that his nostrils were "plugged up" and that a sticky yellow discharge was chronically present. The significant physical findings related to the respiratory tract. A hard granulomatous mass filled the nares, nasopharynx, and the pharynx. In some places the mass was covered by dirty, green, malodorous crusted material which was occasionally expectorated. The voice was hoarse and there was some granulation involving the epiglottis and aryepiglottic folds. The patient complained that the dyspnea interfered with normal activity. There were no other significant laboratory or physical findings.

Biopsy of the lesion demonstrated a typical granuloma with plasma and lymphoid cells. Hyalinization was present. Mikulicz cells were numerous and easily demonstrated. Staining with the Krajian modification of the Gram-Weigert method showed numerous bacilli present, extra- and intracellularly.

The patient has been treated with sulfadiazine, penicillin, and streptomycin. Only the last was really effective against the organism in vitro and caused a decrease in the number of rhinoscleroma bacilli in the throat, but did not affect the lesions after 10 days of treatment with 10 grams of the drug. Deep $x$-ray is being used at the present time.

From the above patient we isolated the scleroma bacillus in twenty consecutive throat cultures on blood agar and E. M. B. agar. The organism grows as a large, mucoid, flowing colony easily picked from the usual throat contaminants after 24 hours of incubation.

On questioning this first patient, we found that his father has similar symptoms, of longer duration. His history follows:

Case 2. L. M., age 37, was essentially well until his nose was broken about 10 years ago. After this his nose became progressively "stuffy" and a thick yellow discharge developed. Rhinoscleroma was diagnosed clinically elsewhere one year after onset and $x$-ray therapy was given. At present, there is adequate airway, but the lining of the nose and pharynx is hard, irregular, and studded with foul smelling, greenish crusts.

Biopsy findings were typical and identical with those of Case 1, including the presence of the small bacilli in stained sections of the tissue. Organisms identical with the scleroma bacillus have been isolated repeatedly in cultures from the nose and throat.

After seeing that the father and son both were suffering from the same disease, we thought that other members of the family might show symptoms of a similar nature. From our discussion with father and son, we knew that they were suffering from vague symptoms associated with the upper respiratory tract. In addition to the son (Case 1) the family consisted of four daughters, a young son age 9 , and the wife of the oldest son (Case 1). The mother of the children has been separated from the family for the past four years and is not available.

The wife of Case 1 and the youngest son are free of symptoms and culturally negative. The four girls seem to show various stages of an infection of the nasopharynx which resembles the "diffuse" type of scleroma described by Szmurlo, and mentioned previously. Their case histories follow :

Case 3. J. M., age 19 (sister of Case 1), is asymptomatic except for frequent "head colds" and a watery nasal discharge which has been chronically present for two years. There is marked atrophy of the inferior and middle turbinates and the nose contains crusts and casts of greenish-yellow matter that has the same foul odor noticed in others of her family.

Biopsy sections show inflammation and the presence of many monocytes. The mucous glands are hyperplastic. The sections stained for bacterial examination were inadequate in this case. Cultures revealed the presence of the scleroma bacillus in the throat and nose.

Case 4. C. M., age 17 (sister of Case 1), is asymptomatic except for a chronic cough which she attributes to a thick, sticky, nasal and postnasal discharge. She had "lobar pneumonia with empyema" three years ago. Recovery followed open drainage. Physical examination of the upper respiratory tract showed marked atrophy of the inferior and middle turbinates with some thickening of the septum superiorly and anteriorly, and the presence of greenish crusts.

Biopsy sections show a diffuse infiltration, granulomatous in type, under the epithelium. Occasional cells of the macrophage variety are present. There are present also large foamy cells not typical of the Mikulicz cell. Bacterial stains show bacilli to be present extracellularly. Repeated cultures of the nose and throat show the presence of the scleroma bacillus.

Case 5. M. M., age 14 (sister of Case 1), complains of increased nasal "stuffiness" of a year and a half duration, with no visible discharge. Physical examination shows almost complete obstruction due to the involvement of the anterior and of the inferior turbinates. The hypertrophied tissue causing obstruction has the appearance of granulation tissue and bleeds easily on touching. There was some crusting due to greenish-yellow purulent exudate.

Biopsy sections show tissue consisting of large strands of hyaline material, and of compact masses of small round cells, mostly the macrophage type. In some areas the tissue appears necrotic with the cells staining faintly. One area shows mucous vacuoles which resemble fat tissue; these are surrounded by round cells of macrophage type. Some of the cells show foamy cytoplasm and some 
are binucleate. No typical Mikulicz cells are seen. No bacterial stain was done. Repeated cultures of the nose and throat showed the presence of the scleroma bacillus.

Case 6. R. M., age 11 (sister of Case 1), is asymptomatic although her sisters complain that she is always clearing her throat. Examination showed considerable hypertrophy of the tonsils. No atrophy, granulation or crusting was present in the nares.

Biopsy of a slightly injected area of the pharyngeal mucosa reveals the presence of stratified and ciliated epithelium below which there are occasional areas of infiltration of macrophages. Small granulomatous lesions are found here and there around capillary vessels. Noticeable is the presence of large cells with peripheral nuclei and foamy cytoplasm. The bacterial stains show bacilli inside the macrophages. The scleroma bacillus was repeatedly isolated from nose and throat cultures.

The last four cases, in our opinion, resemble varying stages of rhinoscleroma. In none of these cases were the symptoms sufficiently severe to result in the patient being referred to a doctor, yet physical examination reveals lesions which at least in three of the cases are suggestive of the disease. In one case, R. M., the youngest sister, there are practically no gross pathologic lesions and yet the microscopic picture in all the cases including the last confirms the existence of the disease syndrome, which, together with the isolation of the scleroma bacillus in each case, gives extremely presumptive evidence that here we have a family in which six members are afflicted with various stages of the disease rhinoscleroma. Serological findings in each of these cases gives further evidence for this contention, although until the complement fixation test, mentioned previously, is standardized, emphasis on the serology must be avoided.

One further case will be presented although it is unrelated to the family mentioned above. This case also gave a positive culture for the scleroma bacillus and a positive complement fixation test.

Case 7. L. C., age 63, female of Mexican descent. The patient was seen first fourteen years ago when she complained of obstruction to her breathing of four months' duration. At that time, "granular pharynx" and hypertrophy of the right mid-turbinate accompanied by postnasal purulent discharge were reported. The patient was not seen again until recently at which time in addition to the previously mentioned symptoms the inferior turbinate on the right also was reported to be hypertrophic and irregular with the appearance of fungating granulation tissue.
Biopsy sections show the presence of shreds of epithelium, dense fibrous strands, and compact masses of round cells. Under high power the cells appear to be mostly plasma cells and lymphocytes, but here and there are seen numerous macrophages with foamy cytoplasm. An occasional large cell with peripheral nucleus and pink cytoplasm is seen. No hyaline globi are visible. Lesions seem to be of the granulomatous type.

\section{CONCLUSION}

Six cases of scleroma in the same family are described showing varying stages of the disease. The etiologic agent is suggested to be the scleroma bacillus first described by Frisch. Its characteristics are given. The incidence of this organism in the normal population has been studied. Suggestive serological findings are reported.

\section{BIBLIOGRAPHY}

1. von Hebra, R., Ueber ein eigenthumliches Neugebilde an der Nose, Rhinosclerom, nebst histologischem Befunde vom Dr. M. Kohn. Wien. med. Wchnschr., 1870, 20, 1.

2. Geber, E., Ueber das Wesen des Rhinosclerom; eine klinischhistologische Studie. Arch. f. Dermat. u. Syph., 1872, 4, 419.

3. Mikulicz, Johann, Ueber das Rhinosclerom. Arch. f. klin. Chir., 1877, 20, 485.

4. Rapp, Cong. Internat. d'oto-rhinolaryng., Madrid, 1932.

5. Szmurlo, J., Anatomicopathology of scleroma. Arch. Otolaryng., 1933, 18, 393.

6. Cunning, D. S., Guerry, Du. P., Scleroma, Arch. of Otolaryng., 1942, 36, 662.

7. Streit, H., Etiology of scleroma. Arch. of Otolaryng., 1933, 18, 392.

8. Lasagna, F., Infective scleroma of the respiratory passages. J. Laryng. and Otol., 1925, 40, 369.

9. von Frisch, A., Zur Aetiologie des Rhinoskleroms. Wien med. Wchnschr., 1882, 32, 969.

10. Perkins, R. G., Relation of the bacillus mucosus capsulatus group to rhinoscleroma and of the various members of the group to one another. J. Infect. Dis., 1904, 1, 241.

11. Gay, F. P., Agents of Disease and Host Resistance. Thomas, Springfield, 1935.

12. Wilson, G. S., Miles, A. A., Topley and Wilson's Principles of Bacteriology and Immunity. 3rd Ed., Baltimore, 1946.

13. Figi, F. A., Thompson, L., Rhinoscleroma. J. A. M. A., 1928, 91, 637.

14. Morris, M. C., and Julianelle, L. A., A biological classification of the bacillus of rhinoscleroma. J. Infect. Dis., 1934, 55, 150.

15. Gonsiorowski, N., and Meisel, H., Sur la biologie de bacilles muqueux encapsules. Comptes Rend. Soc. de Biol., 1929, 100, 298. 
16. Goldzieher, M., and Nueber, E., Untersuchungen über das Rhinosklerom. Centralbl. f. Bakt. (Abt. I), 1909, 51, 121.

17. Brault, J., and Masselot, L., Quelques remarques sur le bacille du rhinosclerome (bacille de Frisch). Arch. de med. exper. et d'anat. path., 1910, 22, 545.

18. Quast, quoted by Figi and Thompson, 1928.

19. Fitzgerald, J. G., A biometrical study of the mucous capsulatus group. J. Inf. Dis., 1914, 15, 268.
20. Prasek, E., and Prica, M., Agglutinationsreaktion bei Rhinosklerom. Centralbl. f. Bakteriol. (Abt. I), 1928, 108, 376.

21. Quoted by Mayer, E., Scleroma of the larynx. Am. J. Med. Sci., 1907, 133, 751.

22. Gerber, P. H., Ueber das Sklerom insbesondere in Ostpreussen. Arch. f. Laryng. u. Rhin., 1900, 10, 347. 